\title{
Dynamics of the laser-induced nanostructuring of thin metal layers: Experiment and theory
}

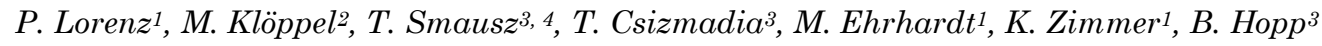 \\ ${ }^{1}$ Leibniz-Institut für Oberflächenmodifizierung e. V., Permoserstraße 15, 04318 Leipzig, Germany \\ 2 Institute of Scientific Computing, Department of Mathematics, TU Dresden, 01062 Dresden, Germany \\ ${ }^{3}$ Department of Optics and Quantum Electronics, University of Szeged, H-6720 Szeged, Dóm tér 9, Hungary \\ ${ }_{4}$ MTA-SZTE Research Group on Photoacoustic Spectroscopy, University of Szeged, H-6720 Szeged, \\ Dóm tér 9, Hungary
}

\begin{abstract}
Nanostructures are of increasing importance in manifold application fields like electronics, optics and beyond. However, the fast and cost-effective production of nanostructures is a big technological challenge for laser machining. One promising approach is the laser irradiation of thin metal layers, which allows the fabrication of metal nanostructures induced by a melting and deformation process. The influence of laser parameters (laser fluence, laser pulse number) on the morphology of the nanopatterned film and the dynamics of the nanostructure formation during excimer laser irradiation of a $20 \mathrm{~nm}$ chromium film on fused silica were studied. The dynamics of nanopatterning, comprising hole and droplet formation, were investigated by time-dependent reflection and transmission measurements as well as time-dependent optical microscopy. The resulting patterns were investigated by optical and scanning electron microscopy (SEM). However, for an optimization of this process a better understanding of the underlying physical phenomena is necessary. Therefore, experimental data of laserinduced nanopatterning were compared with results of physical simulations that consider the heat equation (laser-solid interaction including melting and evaporation) and the Navier-Stokes equation (deformation processes of the molten phase). The simulations, making use of laser fluence-dependent effective material parameters (surface tension and viscosity), are in good agreement with the experimental results.
\end{abstract}

Keywords: excimer laser, nanostructuring, metal layer, simulation, droplet formation

\section{Introduction}

The fast and efficient fabrication of well-defined nanostructures is of scientific as well as of practical interest, targeting a growing market as a result of the ongoing miniaturization efforts in electronics and other application fields. However, the development of easy and cost-effective nanostructure technologies is a big challenge. Laserbased structuring methods have an outstanding potential for the fast and direct fabrication (no further fabrication steps are necessary) of nanostructures. In particular, self-organization effects such as fs laser-induced ripple structures [1-4], fs laser metal nanostructuring [5-7], and ns laser nanostructuring of metal layers [8-10] are of high interest. Furthermore, laser ablation [11-14] and laser etching [15-18] methods allow the fabrication of vertical nm-precision and lateral sub- $\mu \mathrm{m}$-precision structures in different solids: metals, semiconductors and dielectrics.

The laser-induced nanostructuring of thin metal layers on dielectric surfaces due to laser-induced melting within a short time is well-known [19-25]. The irradiation of the metal film on dielectric surfaces with high laser fluences results in an ablation process of the metal layer and in a modification of the dielectric surfaces [23-25]. The nanosecond irradiation at low laser fluences causes a melting and deformation process of the metal layer and, finally, a droplet formation process [8-10, 26, 27]. The nanostructuring process involves different physical processes: photon-solid interaction [21, 22], energy dissipation including phase transition, and solid-solid interaction (formation in unstable thin liquid films and other results) [10, 28-34]. In a good approximation the laser-induced melting deformation process can be physically described by a heat equation (laser-solid interaction) and some kind of Navier-Stokes equation (mass transport in liquid) [26].

The most studies of laser-induced nanostructuring by pulsed laser melting of thin films focus on the morphology of the achieved metal nanostructures and investigate the influence of fabrication process parameters such as laser fluence, pulse number, or metal film thickness. These morphological characteristics are discussed in relation to the mechanism and results from process simulations. Despite these studies, the mechanism of this laser-induced nanopatterning process is still in discussion. Therefore, for the identification of the underlying physical phenomena extended studies with a different focus are essential. Until now the dynamics of these processes are 
not experimentally studied in detail, but by providing such results more insight into the mechanism can be expected.

Dynamic processes of laser ablation and pattern formation can be studied by pump probe experiments [35].

The aim of this study is to analyse the dynamics of the laser-induced deformation process of metal layers by time-dependent reflection and transmission measurements as well as time-dependent optical imaging of the surface by a pump-probe set-up.

This paper is mainly focussed on the basic parameters of the melting deformation process like the life time of the liquid phase and the velocity of the deformation process where the experimentally estimated parameters were compared with theoretically predicted values.

\section{Experimental details}

The time-dependent melting deformation of $20 \mathrm{~nm}$ chromium magnetron-sputtered on fused silica (double-sided polished, surface roughness $0.25 \mathrm{~nm}$ rms, peak-to-valley value: $1.2 \mathrm{~nm}$, thickness $380 \mu \mathrm{m}$ ) was measured applying a pump-probe set-up (see Fig. 1). The chromium layer exhibits a surface roughness of $\sim 0.3 \mathrm{~nm}$ and a peak-to-valley value of $\sim 2 \mathrm{~nm}$.

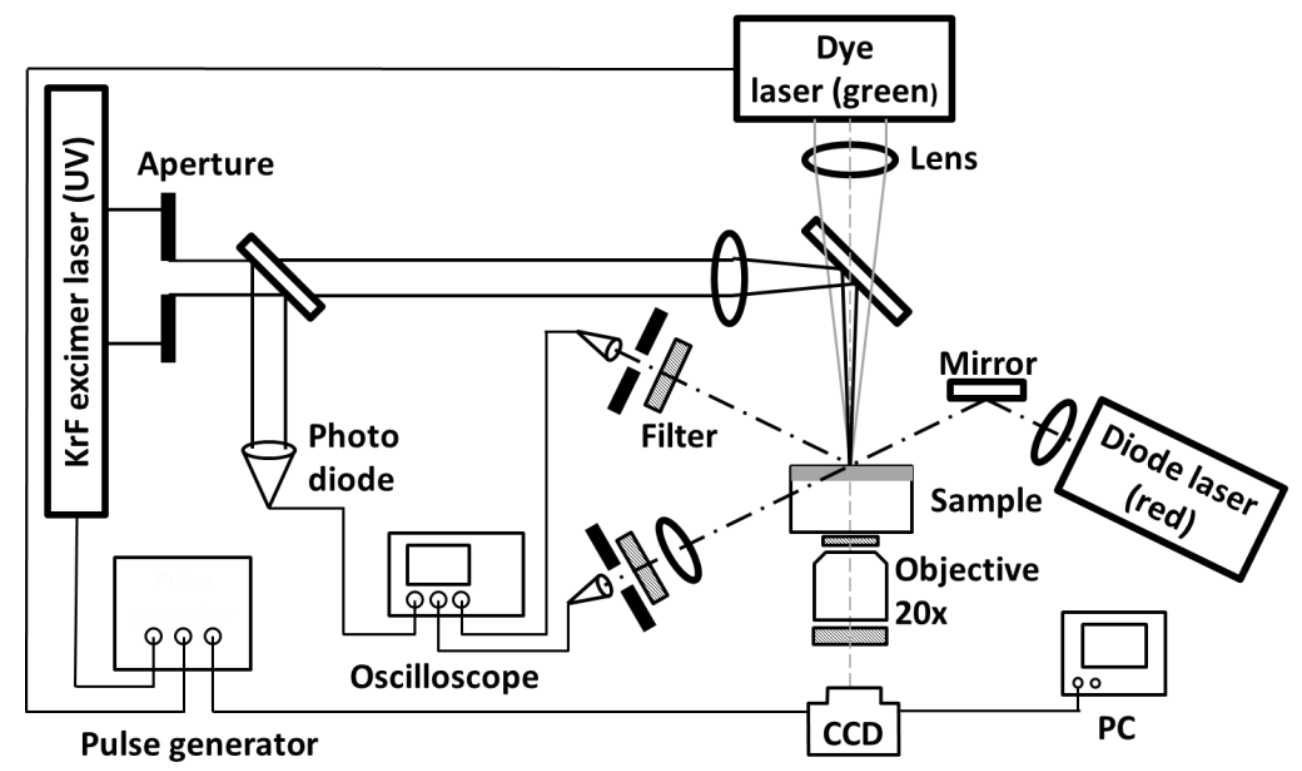

Fig. 1 Schematic illustration of the experimental set-up.

The melting process was induced by a $\mathrm{KrF}$ excimer laser with a wavelength of $\lambda=248 \mathrm{~nm}$ and a pulse duration of $\Delta t_{p}=25 \mathrm{~ns}$. A homogeneous part of the laser was selected by an aperture and imaged to the sample surfaces by a lens to an area of $\sim 1 \mathrm{~mm}^{2}$. By changing the pulse energy of the laser an excimer laser fluence of up to $7 \mathrm{~J} / \mathrm{cm}^{2}$ can be achieved. In addition, the sample surfaces were irradiated with a red $\mathrm{cw}$ diode laser $(\lambda=660 \mathrm{~nm}$, $\mathrm{P}=130 \mathrm{~mW}$ ) and the time-dependent transmitted and reflected laser beam intensity was measured with photodiodes (rise time $\Delta \mathrm{t}_{\mathrm{ph}}=1 \mathrm{~ns}$ ) and a $1 \mathrm{GHz}$ oscilloscope. The experimental set-up allows the recording of the transmission and reflection signals with a temporal resolution of better than $3 \mathrm{~ns}$ and a precision of less than $2 \mathrm{~ns}$ to the actual excimer laser pulse, which was used as reference signal for the experiments and the graphs $(t=0$, e.g. see Fig. 2 b). An aperture/filter combination in front of the photodiodes was used to reduce the influence of the UV and green laser onto the measured reflection and transmission. For the direct time-dependent optical imaging of the metallic surface structure the sample was irradiated by an electronically delayed $\mathrm{N}_{2^{-}}$ pumped green dye laser (Coumarin $153, \lambda=543 \mathrm{~nm}, \Delta \mathrm{t}_{\mathrm{p}}=1 \mathrm{~ns}, \mathrm{E}=1 \mathrm{~mJ}$ ). The sample surface structures were imaged in transmission and in bright field mode by a 20x objective that was optically corrected to the fused silica sample thickness on an external triggered CCD camera with a gate time of less than $1 \mathrm{~ms}$. The image quality was improved by a band pass filter system for blocking UV and red laser radiation. The time delay between the KrF excimer laser, the dye laser, and the gate signal of the camera was adjusted by a freely programmable pulse and delay generator. The pulsed dye (green) laser with a laser fluence of approx. $1 \mathrm{~mJ} / \mathrm{cm}^{2}$ may influence the sample heating slightly but only after the probe pulse (the dye laser typically emitted $20 \mu \mathrm{s}$ after the excimer laser); hence, the image shows only morphological alterations of the samples due to the excimer laser pulse. Furthermore, a crucial influence of red cw laser radiation on the metal layer/dielectric system was excluded by a long-time $(\mathrm{t}>10 \mathrm{~s})$ analysis of the reflection and transmission signals, which yielded that the red cw laser radiation does not influence the optical signal. This means that a red $\mathrm{cw}$ laser-induced distinct increasing of the 
surface temperature and a modification of the metal layer can almost be excluded. The resultant structures were analysed by optical and scanning electron microscopy (SEM).

\section{Experimental results}

The $20 \mathrm{~nm} \mathrm{Cr} /$ fused silica samples were irradiated with a $\mathrm{KrF}$ excimer laser applying different laser fluences and numbers of laser pulses. Within the experiments the laser irradiation was especially performed with high and low laser fluences where the maximum expedient laser fluences are defined by the ablation threshold. For the complete removal of the chromium layer with 1 pulse $\Phi_{\mathrm{th}}{ }^{\text {eva }}(\mathrm{N}=1) \sim 2 \mathrm{~J} / \mathrm{cm}^{2}$ are necessary where this value decreased at increasing number of laser pulses.

The experimental findings was separated into a high and low fluence part, where the for the high fluence part the results for a laser fluence of $750 \mathrm{~mJ} / \mathrm{cm}^{2}$ and for the low laser fluence part of $480 \mathrm{~mJ} / \mathrm{cm}^{2}$ are exemplary presented.

\subsection{High fluence range}
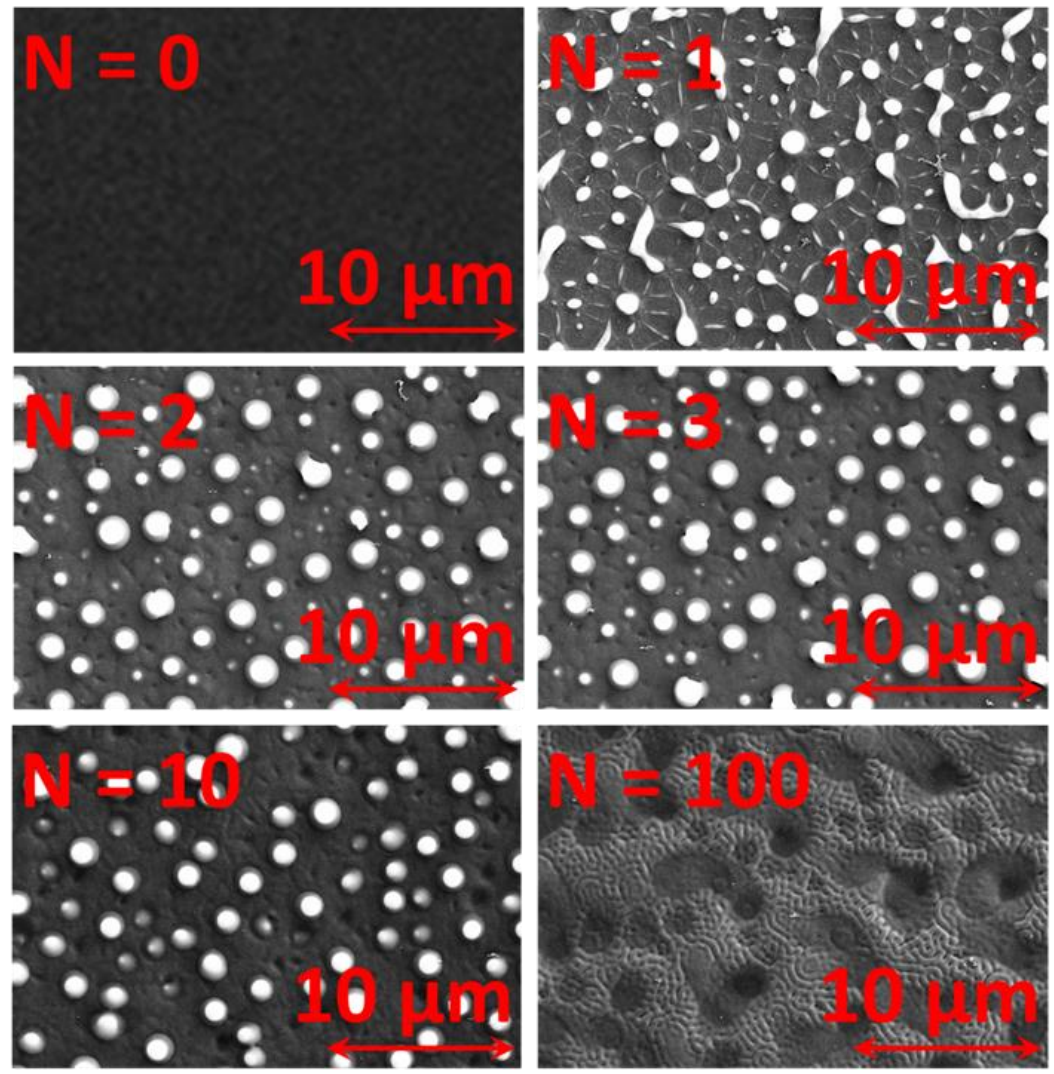

(a)

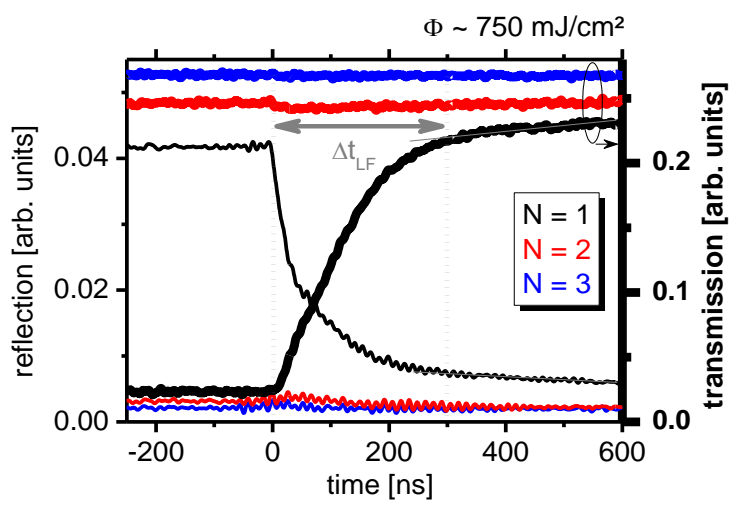

(b) 
Fig. 2 (a) SEM image of irradiated $20 \mathrm{~nm}$ Cr/fused silica at $\Phi=(750 \pm 50) \mathrm{mJ} / \mathrm{cm}^{2}$ and $N=0,1,2,3,10,100$ (measured at different samples).

(b) Time-dependent reflection and transmission for different laser pulses $N=1-3$ at $\Phi=(750 \pm 50) \mathrm{mJ} / \mathrm{cm}^{2}(\mathrm{the}$ reflection and transmission signals were smoothed by a low pass filter).

In Fig. 2 (a) the SEM images of chromium-covered fused silica irradiated with different laser pulse numbers at a laser fluence of $\Phi=(750 \pm 50) \mathrm{mJ} / \mathrm{cm}^{2}$ are shown.

The irradiation of the homogeneous covered fused silica (see Fig. 2 (a) at $\mathrm{N}=0$ ) results in a distinct modification of the topography of the metal layer already at the first pulse (see Fig. 2 (a) at $\mathrm{N}=1$ ), i.e., in surface roughening and an incomplete droplet formation. The interaction of the second laser pulse with this modified, roughened chromium surface causes a complete droplet formation (see Fig. 2 (a) $\mathrm{N}=2$ ). From this SEM image at $\mathrm{N}=2$ a droplet diameter of $(0.9 \pm 0.4) \mu \mathrm{m}$ can be determined. Further laser pulses implicate much less modification of the droplets (see Fig. 2 (a) $\mathrm{N}=3$ ).

The optical images are limited, due to the low optical resolution $(1$ pixel $=1.25 \mu \mathrm{m})$ in comparison to the droplets size. However, the optical measurement allows to display the variation of the chromium on the fused silica (especially the hole formation process, see Fig. 3). A better way for the optical study of the modification of the metal layer is the analysis of the reflection and transmission signals.

The measured time-dependent reflection and transmission light of the $\mathrm{cw}$ probe laser at the first, second, and third pulse is shown in Fig. 2 (b). The SEM of the samples suggests that the main variation of the transmission and reflection signals should occur at the first laser pulse. This assumption is confirmed by the time-dependent intensity measurements of the reflected and transmitted red probe laser light. The first laser pulse with a laser fluence of $(750 \pm 50) \mathrm{mJ} / \mathrm{cm}^{2}$ induces a distinct reduction of the reflection signal intensity (see Fig. 2 (b) thin black line) and an increase in the transmission signal (see Fig. 2 (b) thick black line). The temporal behaviour of the intensity change of the transmitted and the reflected signals is complementary and can be characterized by a time constant that describes the time for the morphological changes of the thin film during the liquid phase. Hence, the transformation time $\Delta \mathrm{t}_{\mathrm{LF}}{ }^{\exp }$ that mainly determines the modification time of the metal film can be deducted from the transmission and reflection signals and was determined for the applied laser fluence to be $\Delta \mathrm{t}_{\mathrm{LF}}{ }^{\text {exp }}=(300 \pm 30) \mathrm{ns}$ (see Fig. $2(\mathrm{~b})$ ). These experimentally measured transformation times of the thin film $\Delta \mathrm{t}_{\mathrm{LF}}{ }^{\mathrm{exp}}$ are shown in Fig. 4 in dependence on the laser fluence $\Phi$.

Furthermore, the second pulse induces a small reduction of the reflection and a small increase in the transmission signal. This small variation can be explained by the completion of the droplet formation process (see SEM image Fig. 2 (a) (i) $\mathrm{N}=1$ and $\mathrm{N}=2$ ).

In contrast to the SEM and optical images, which appear similar, the transmission and reflection signals reveal small variations of the surface at $\mathrm{N}=3$; in particular, the reflection signal is almost constant and the transmission signal increases at $\sim 8 \%$ in comparison to the signal after the second pulse. The further increase of the transmission signal can most likely be explained by a partial evaporation of the remaining surface modifications of the already exposed fused silica, e.g. thin chromium debris or modified fused silica. At higher pulse numbers (see Fig. 2 (a) $\mathrm{N}=10$ ) a reduction of the droplet density most likely induced by an evaporation process of the chromium can be found. In contrast to the experimental found $\Phi_{\mathrm{th}}{ }^{\text {eva }}(\mathrm{N}=1)$ the theoretical ablation threshold $\Phi_{\text {th }}{ }^{{ }^{e v a *}}=\rho \cdot \Delta z / R \cdot\left(c \cdot\left(T_{\text {eva }}-293.15 \mathrm{~K}\right)+\Delta H_{\text {eva }}\right)(\Delta z$ : metal layer thickness, material parameter see section 4.) is with $278 \mathrm{~mJ} / \mathrm{cm}^{2}$ defined smaller than the experimental found value. That means, a partial evaporation of the chromium at higher laser fluences is most likely. Prolonged laser irradiation finally results in a complete evaporation of the chromium and in a nanostructuring of the fused silica surface.

\subsection{Low fluence range}

The droplet formation process requires a substantial higher number of laser pulses at low laser fluences. In Fig. 3 the optical microscopic (a) and the SEM images (b) are shown for different numbers of laser pulses with $\Phi=(480 \pm 40) \mathrm{mJ} / \mathrm{cm}^{2}$. In particular, the SEM measurements allow the monitoring of the stepwise development of the geometrical surface features (see Fig. 3 (b)). The irradiation of the metal layer results in the formation of hole structures within the metal film where the hole radius increases with increasing laser pulse number. In addition, further laser pulses cause the formation of new holes.

The maximum hole size increases with the pulse number as shown in Fig. 3 (c) (right) and the approximated value is $d_{\max }=(8.3 \pm 0.5) \mu \mathrm{m}$ at the applied laser fluence. The limitation of the hole size is given by the dynamic of the hole development with the hole size density that increases with the pulse number and the individual hole size that is limited by the geometrical confinement of the hole size growth due to the collision of the hole rims. Hence, the pulse number of the encounter of two separated holes locally varies and can be found in the images for the first time at $\mathrm{N}=5$. After coalescence, the hole droplets are formed from the hole rims. An average droplet size of $(0.8 \pm 0.3) \mu \mathrm{m}$ at $\mathrm{N}=20$ can be estimated from the SEM images. 
Basically, the same behaviour of the hole development can be observed during optical microscopy (see Fig. 3 (a)). The measurement of the hole sizes is not expedient due to the limited optical resolution and diffraction effects.

However, the measured transmitted light distribution allows the detection of holes formation and especially the estimation of the ratio of uncovered to covered surfaces. At the first pulse the formation of small holes can be detected. Further irradiation results in the growth of the existing and the formation of new holes and droplets.

Therefore, the ratio of bright (uncovered surface) and dark (covered surface) regions was calculated from the histogram of the greyscale images and is shown in Fig. 3 (a) (left).

The transmission and reflection signals were measured, too. Similar to the high laser fluence results (see Fig. 2 (b)), low laser fluence irradiation results in an increase of the transmission and in a reduction of the reflection signal during the time of laser-induced surface modification. The transmission signal intensity, which was measured after the resolidification of excimer laser-induced molten film ( $\mathrm{t}>$ liquid phase time $\Delta \mathrm{t}_{\mathrm{LF}}$ ), is shown in Fig. 3 (c) (left).

The transmission signal $\mathrm{T}$ only slightly increases with increasing laser pulse number for $\mathrm{N} \leq 8$. For high pulse numbers $(\mathrm{N}>8)$ the slope of the transmission $\mathrm{T}(\mathrm{N})$ is noticeably higher. The comparison of the transmission signal with the uncovered surface ratio (see Fig. 3 (a) (left)) suggests that the transmission signal increase can be explained by the dewetting process of the chromium. The estimation of the liquid phase life time $\Delta t_{L F}$ at low laser fluences that was performed similar to the evaluation of the transmission and reflection measurements at high laser fluences (see Fig. 2 (b)) gives distinct smaller time values (see Fig. 4).

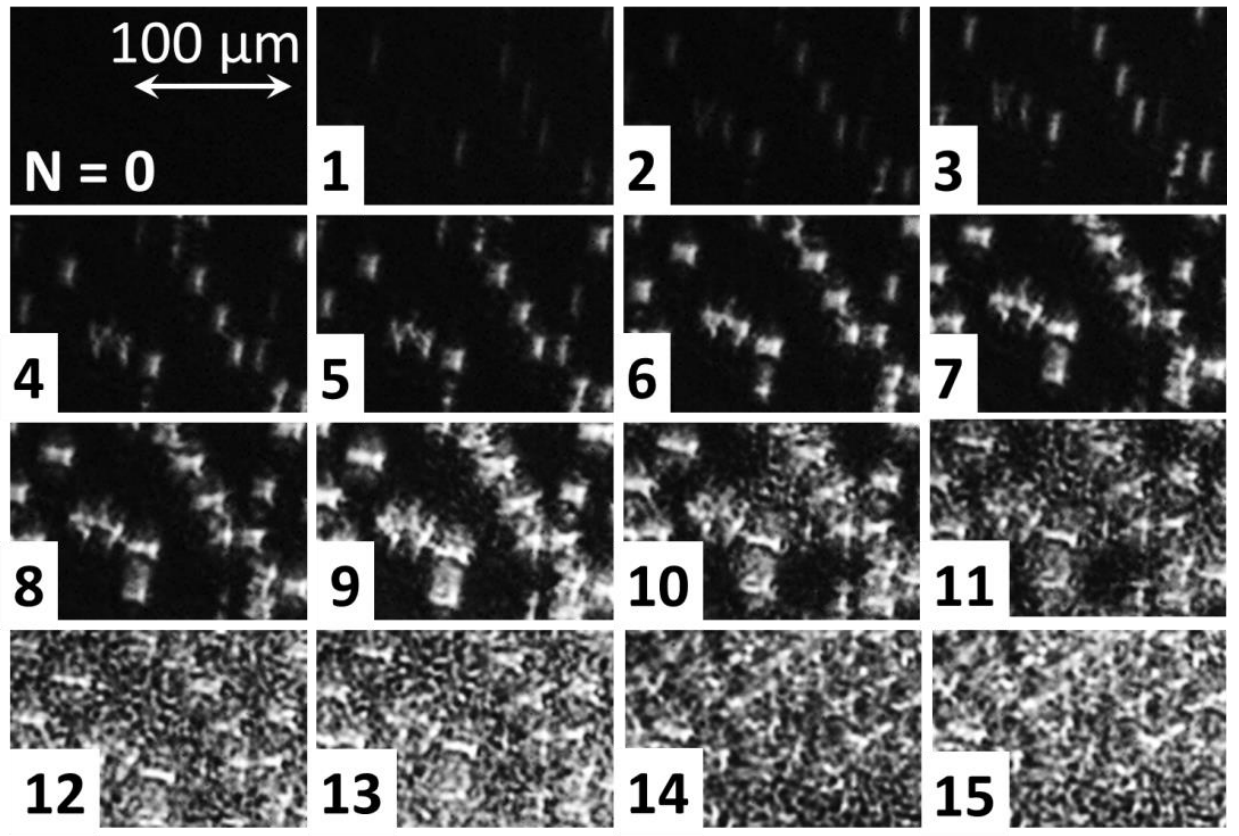

(a)
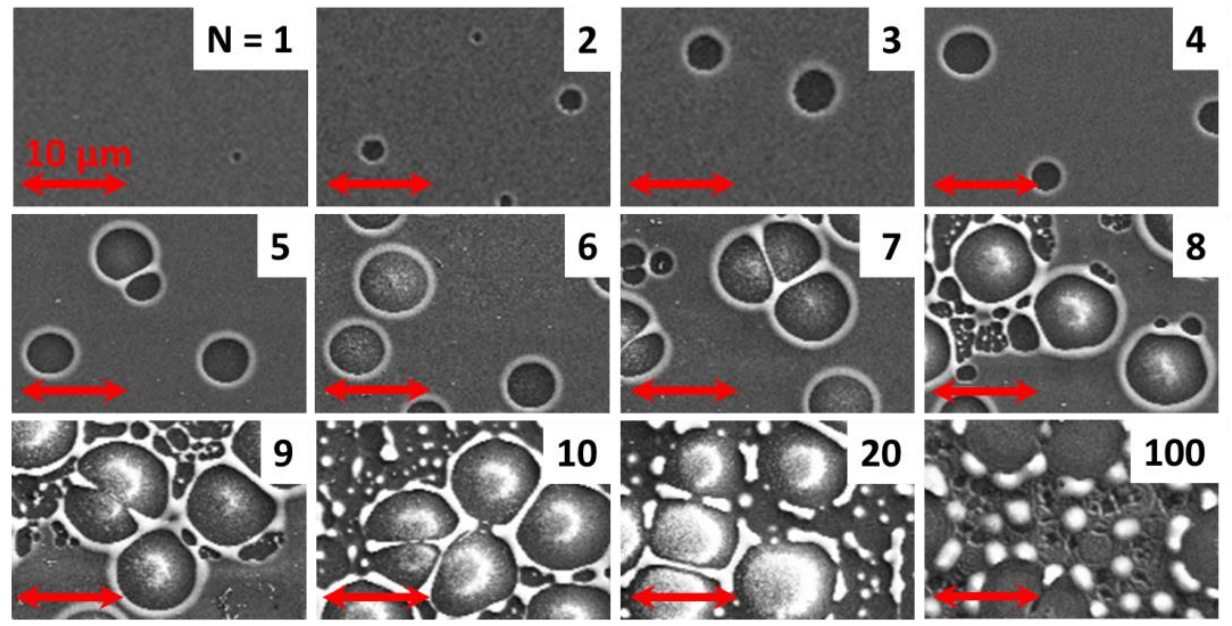

(b) 

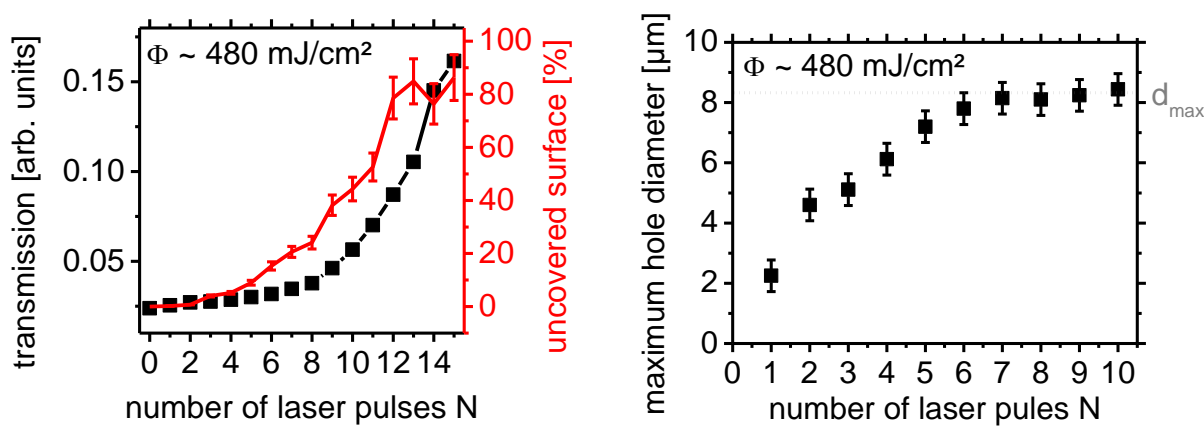

(c)

Fig. 3 (a) Optical microscopic image with different laser pulses $N=0-15$ measured $20 \mu$ s after the laser pulse (b) SEM image of the irradiated $20 \mathrm{~nm}$ Cr/fused silica system $\left(\Phi=(480 \pm 40) \mathrm{mJ} / \mathrm{cm}^{2}, N=1-100\right)$.

(c) (left) Transmission signal and estimated uncovered surface (in percent relative to the complete surface), based on the optical measurements at different laser pulses $N=0-15$, (right) maximum hole radius at different laser pulses $N=1-10$.

The measured transformation time $\Delta \mathrm{t}_{\mathrm{LF}}$ in dependence on the laser fluence is shown in Fig. 4 (blue line) and can be fitted by a linear function very well:

$$
\Delta t_{L F}^{\exp }=m^{*}\left(\Phi-\Phi_{t h, m}\right)
$$

with a slope coefficient of $\mathrm{m}=0.63 \mathrm{~ns} /\left(\mathrm{mJ} / \mathrm{cm}^{2}\right)$ and melting threshold $\Phi_{\mathrm{th}, \mathrm{m}}=212 \mathrm{~mJ} / \mathrm{cm}^{2}$.

\section{Theory}

To explain the laser-induced formation of metal nanostructures on fused silica, the mechanism must be considered and the involved processes should be simulated for comparison with the experimental results. At least two dominating processes must be considered for the physical discussion of the mechanism of the structure formation processes for laser irradiation of thin films: (i) laser absorption, materials heating, and heat dissipation including materials melting (heat equation) and (ii) mass transport in the liquid [26]. It is clear that both processes occur at the same time and affect each other; hence, they are strictly coupled. Supported by the experiments that clearly show a particular liquid phase life time at the end and after the laser pulse, the separation of the processes is assumed to simplify the calculations and to reveal straightforward correlations. Therefore, in this study the laser-induced nanostructuring was simulated under the assumption that the physical processes (laser-solid-interaction, liquid film interaction) can be regarded as decoupled.

(i) Laser absorption and heat dissipation (heat equation)

The absorption of the laser radiation leads to the heating of the chromium film and, due to heat dissipation, to the heating of the fused silica near the surface. The laser-induced temperature distribution can be calculated by solving

$$
\begin{aligned}
& \rho_{M, S} \cdot c_{p ; M, S} \cdot \dot{T}\left(t, \vec{r}_{s}\right)-\nabla\left(\kappa_{M, S} \cdot \nabla T\left(t, \vec{r}_{s}\right)\right)=Q\left(t, \vec{r}_{s}\right) \\
& =\left\{\begin{array}{c}
\frac{\Phi\left(t, \vec{r}_{s}\right) \cdot\left(1-R_{o p t}\right)}{\Delta t_{p}} \cdot \alpha \cdot \exp (-\alpha \cdot z) \text { at } M \\
0 \text { at } S
\end{array}\right.
\end{aligned}
$$

with T: temperature, Q: laser-induced heat source, $r_{s}=r_{s}(x, y, z)$ : spatial coordinate, $\rho$ : density (M: metal layer, $\mathrm{S}$ : fused silica), $\mathrm{c}_{\mathrm{p}}$ : effective heat capacity at constant pressure including the effect of the phase transition, $\kappa$ : thermal conductivity, $\alpha$ : absorption coefficient $(1 / \alpha=10.42 \mathrm{~nm}[24]), \Phi$ : laser fluence (a lateral homogeneous laser radiation was assumed), $\mathrm{R}_{\mathrm{opt}}$ : reflectivity (a constant reflectivity of 0.4 was assumed), $\Delta \mathrm{t}_{\mathrm{p}}$ : laser pulse duration (based on the measured laser pulse - time dependency a $20 \mathrm{~ns}$ long rectangular laser beam was assumed.). The irradiation at a sufficient laser fluence results in a partial melting and partial evaporation of the metal layer and of the fused silica surface, where the melting and evaporation enthalpy was regarded (see Tab. 1). The effect of the melting and evaporation enthalpy was considered by a variation of the effective heat 
capacity $c_{p}$ near by the melting and evaporation temperature. A detailed description of the phase transition in the heat equation can be found in e.g. [36].

\begin{tabular}{|c|c|c|}
\hline & $\mathrm{Cr}$ & $\mathrm{SiO}_{2}$ \\
\hline Melting enthalpy $\Delta \mathrm{H}_{\mathrm{m}}$ & $325 \mathrm{~kJ} / \mathrm{kg}$ & $142 \mathrm{~kJ} / \mathrm{kg}$ \\
\hline Evaporation enthalpy $\Delta \mathrm{H}_{\mathrm{eva}}$ & $6620 \mathrm{~kJ} / \mathrm{kg}$ & $4340 \mathrm{~kJ} / \mathrm{kg}$ \\
\hline Melting temperature $\mathrm{T}_{\mathrm{m}}$ & $2180 \mathrm{~K}$ & $1988 \mathrm{~K}$ \\
\hline Evaporation temperature $\mathrm{T}_{\mathrm{eva}}$ & $2755 \mathrm{~K}$ & $2503 \mathrm{~K}$ \\
\hline Tab. 1: Summary of the assumed melting and evaporation enthalpies as well as the temperatures of chromium and fused silica [37,38].
\end{tabular}

Eq. 2 was numerically solved using the finite element method (FEM) applying Comsol 4.1 [23]. The material parameters were taken from the material database of Comsol 4.1. Further information about the thermodynamic model used for the simulation can be found in Ref. [23]. The solution of Eq. 1 allows the estimation of the timedependent temperature distribution (see Fig. 4 a) and, further, the estimation of the liquid phase life time of the metal layer $\Delta \mathrm{t}_{\mathrm{LF}}{ }^{C r}$ in regard to the melting temperature $\mathrm{T}_{\mathrm{m}}{ }^{\mathrm{Cr}}$ (see Tab. 1). The $\Delta \mathrm{t}_{\mathrm{LF}}{ }^{\text {exp }}$ is defined by the time range $\Delta \mathrm{t}=\mathrm{t}_{\max }-\mathrm{t}_{\min }$ with the condition $\mathrm{T}(\mathrm{x}=0, \mathrm{z}=0, \mathrm{t}) \geq \mathrm{T}_{\mathrm{m}}{ }^{\mathrm{Cr}}$ with $\mathrm{t}_{\min } \leq \mathrm{t} \leq \mathrm{t}_{\max }(\mathrm{z}=0$ : metal layer - fused silica interface) (see Fig. 4 (a)). The calculated liquid phase life time depends on the laser fluence and is shown in Fig. 4 (b) (red/white dots) for a $20 \mathrm{~nm}$ chromium layer on fused silica. Furthermore, the liquid phase life time can be analytically estimated by second-degree polynomial:

$$
\Delta t_{L F}^{t h}(\Phi)=\left\{\begin{array}{c}
a+b \cdot \Phi+c \cdot \Phi^{2} \text { for } \Phi \geq \Phi_{t h} \\
0 \text { elsewhere }
\end{array}\right.
$$

with $\mathrm{a}=3.6 \mathrm{~ns}, \quad \mathrm{~b}=-0.05029 \mathrm{~ns} /\left(\mathrm{mJ} / \mathrm{cm}^{2}\right)$, and $\mathrm{c}=0.00107 \mathrm{~ns} /\left(\mathrm{mJ} / \mathrm{cm}^{2}\right)^{2}$ (see Fig. 4 (red line) with a determination coefficient of $\mathrm{R}^{2}=0.992$.

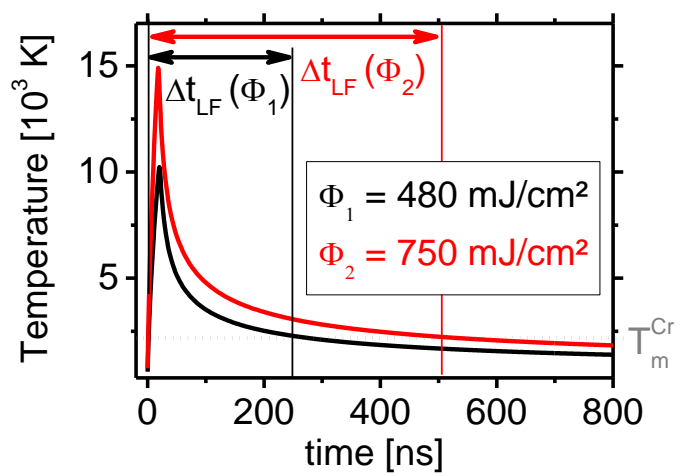

(a)

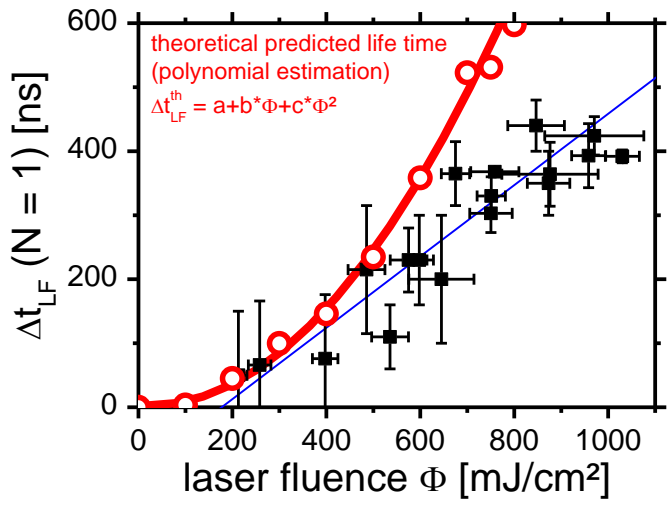

(b)

Fig. 4 (a) Estimated temperature - time dependency at $\Phi=480 \mathrm{~mJ} / \mathrm{cm}^{2}$ using Eq. 2 (black line) and at $\Phi=750 \mathrm{~mJ} / \mathrm{cm}^{2}$ (red line), (grey dotted line) melting temperature of the chromium layer, (black and red arrows) liquid phase life time $\Delta t_{L F}$.

(b) black square: measured transformation time dependent on the laser fluence $\Phi$ (see Fig. 2 (b)),

thin blue line: linear estimation of the measured transformation time dependency $\left.\Delta t^{e x p}{ }_{L F}=m \cdot\left(\Phi-\Phi_{t h}\right)\right)$ with $m=0.63 \mathrm{~ns} /\left(\mathrm{mJ} / \mathrm{cm}^{2}\right)$, and $\Phi_{t h, m}=212 \mathrm{~mJ} / \mathrm{cm}^{2}$, (red/white dots) thermodynamically based prediction of the life time (see section 4(i)), (red line) polynomial estimation of the thermodynamically predicted estimation $\Delta t^{\text {th }}{ }_{L F}=a+b \cdot \Phi+c \cdot \Phi^{2}$ with $a=3.6 \mathrm{~ns}, \quad b=-0.05029 \mathrm{~ns} /\left(\mathrm{mJ} / \mathrm{cm}^{2}\right)$, $c=0.00107 \mathrm{~ns} /\left(\mathrm{mJ} / \mathrm{cm}^{2}\right)$. The ablation threshold for single pulse is $\sim 2 \mathrm{~J} / \mathrm{cm}^{2}$.

(ii) Mass transport in the liquid (Navier-Stokes equation)

The laser-induced liquid metal layer exhibits a restructuring of the molten material induced by the surface energy of the liquid film. The restructuring process induces a dewetting process: it starts with a hole formation process and finally results in a droplet formation (see Fig. 2 and 3). This behaviour can be described by a kind of NavierStokes equation [28]:

$$
\frac{\partial h}{\partial t^{\#}}+\nabla \cdot\left(h^{3} \cdot \nabla\left(\nabla^{2} h\right)\right)-\nabla \cdot\left(h^{3} \cdot \Phi_{h} \cdot \nabla h\right)=0
$$

with $\mathrm{h}=\mathrm{h}\left(\mathrm{x}^{\#}, \mathrm{y}^{\#}, \mathrm{t}^{\#}\right)$ : non-dimensional local film thickness scaled by mean thickness $\Delta \mathrm{z}, \mathrm{x}^{\#}, \mathrm{y}^{\#}$ : lateral coordinates scaled by the characteristic length $\Gamma$ (see Eq. 5a), the time is normalized by $\Psi$ (see Eq. 5b) with 
$\gamma$ : surface tension, $\mu$ : viscosity, A: Hamaker constant, and $\Phi_{h}=\left(\frac{2 \pi \cdot \Delta z^{2}}{|A|}\right) \cdot \frac{\partial^{2} G}{\partial h^{2}}$ : excess intermolecular interactions, $G=-\frac{A}{12 \pi \cdot h^{2}}+S^{P} \cdot \exp \left(-\frac{h}{l}\right)$ : free energy, $S^{\mathrm{P}}$ : non-van-der-Waals attraction, 1: correlation length.

$$
t=\frac{12 \pi^{2} \mu \cdot \gamma}{A^{2}} \cdot \Delta z^{5} \cdot t^{*}=\Gamma \cdot t^{*} \quad \text { (5a) } \quad r=\sqrt{\frac{2 \pi \cdot \gamma}{A}} \cdot \Delta z^{2} \cdot r^{*}=\Psi \cdot r^{*}
$$

Eq. 4 allows a description of the time-dependent topographic variations of the molten metal layer. This equation is solved using central difference in space and implicit backward difference formulas in time. For a more detailed description of the process see [26]. Eq. 4 allows the estimation of the dewetting process of $20 \mathrm{~nm}$ chromium on fused silica under the assumption of a Hamaker parameter of $\mathrm{A} \approx 5.7 \cdot 10^{-19} \mathrm{~J}$, correlation length $1 \approx 0.0137 \mathrm{~nm}$ $[33,39]$. In Fig. 5 the calculated hole diameter - time dependency is shown where the presented values are normalized; see Eq. 5a and Eq. 5b. The hole formation process was calculated up to the complete relaxation of the opening process (the dewetting and opening process is completed with the droplet formation process).

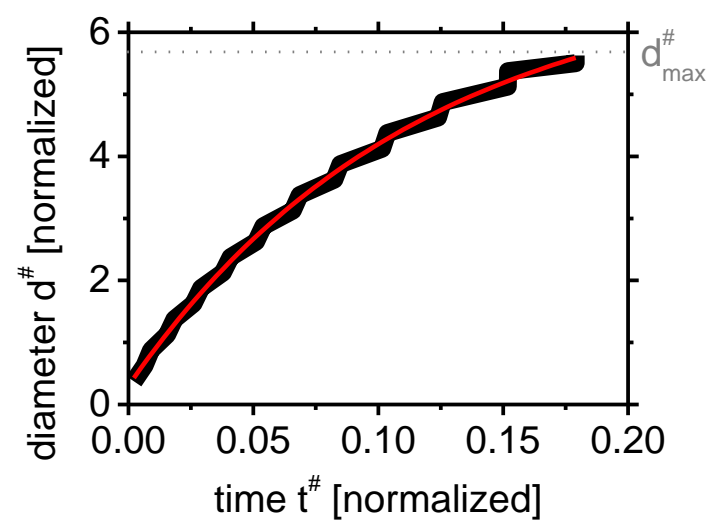

Fig. 5 (black line) Estimated normalized hole diameter normalized time dependency using Eq. 4,

(red line) analytic approximation of the theoretically predicted behaviour using Eq. 6 a (left side).

The estimated $\mathrm{d}^{\#}\left(\mathrm{t}^{\#}\right)$-dependency can be described by an analytical function very well, see Eq. 6a (left side) and Fig. 5, with a coefficient of determination $R^{2}=0.9988$ and the parameter: $A^{\#}=6.985, B^{\#}=-1.659 \cdot 10^{14}$, $\mathrm{t}_{0}^{\#}=-3.529, \tau^{\#}=0.1144$.

$$
\left.d^{\#}\left(t^{\#}\right)=A^{\#}+\frac{B^{\#}}{1+\exp \left(\left(t^{\#}-t_{0}^{\#}\right) / \tau^{\#}\right)} \Rightarrow d_{h}\left(\Gamma t^{\#}\right)=\Psi \cdot\left(A^{\#}+\frac{B^{\#}}{1+\exp \left(\Gamma \cdot\left(t^{\#}-t_{0}^{\#}\right) /\left(\Gamma \cdot \tau^{\#}\right)\right.}\right)\right)
$$

Under the assumption of the normalization, see Eq. 5a, b, the analytical function Eq. 6a (left side) allows the prediction of the hole diameter dependency on the time, see Eq. 6a (right side).

Furthermore, the analytical prediction of the $d_{h}(t)$ - dependency allows the estimation of the hole diameter dependency on the laser parameters taking into account the liquid phase life time - laser fluence dependency (see Fig. 4). It is: 


$$
d_{h}(\Phi, N) \approx A+\frac{B}{1+\exp \left(\left(N \cdot \Delta t_{L F}(\Phi)-t_{0}\right) / \tau\right)}
$$

with $\mathrm{A}=\Psi \cdot \mathrm{A}^{\#}, \mathrm{~B}=\Psi \cdot \mathrm{B}^{\#}, \mathrm{t}_{0}=\Gamma \cdot \mathrm{t}_{0}^{\#}, \tau=\Gamma \cdot \tau^{\#}$.

\section{Discussion}

The laser irradiation of the $20 \mathrm{~nm}$ chromium/fused silica system results in a transformation of the thin metal layer. The laser treatment process results in a melting of the chromium layer and the surface tension leads to a dewetting and a droplet formation process, respectively (see Fig. 2 and 3) for laser fluences higher than the melting threshold. The theoretical melting threshold $\Phi_{\mathrm{th}}{ }^{\mathrm{m}}=\rho \cdot \Delta \mathrm{z} / \mathrm{R} \cdot\left(\mathrm{c} \cdot\left(\mathrm{T}_{\mathrm{m}}-293.15 \mathrm{~K}\right)+\Delta \mathrm{H}_{\mathrm{m}}\right)$ based on the material parameter presented in the previous chapter is $42 \mathrm{~mJ} / \mathrm{cm}^{2}(\Delta \mathrm{z}$ : metal layer thickness).

The dewetting process starts with a hole formation (see Fig. 3). This process is described, e.g. for a $10 \mathrm{~nm}$ chromium layer on fused silica, in [26]. Amongst others, for the $10 \mathrm{~nm}$ Cr layer it was shown that the hole radius increases with increasing laser fluence and number of laser pulses. The nucleation of holes may occur due to local variations of film properties like the thickness of the film (roughness) due to the film grains, the strain of the film due to the film deposition, the heating, or the film composition. Further hole nucleation processes can result from bubble formation at the film $\mathrm{SiO}_{2}$-substrate interface due to evaporation or decomposition. Materials at the interface are different in structure and composition and are often more fragile. Therefore, laser heating can induce small bubbles that locally alter the film thickness and the thermal conductivity that may serve hole nucleation sites. Furthermore, the noise of the laser beam can also contribute to lateral temperature variations and induce hole nucleation. In addition, the metal layer deformation process can be influenced by tension waves. Besides the microscopic analyses of the resultant structures also the time-dependent reflection $\mathrm{R}$ and transmission signal $\mathrm{T}$ of the $\mathrm{Cr} / \mathrm{SiO}_{2}$ system were measured during and after the laser treatment. The analysis of the transmission and reflection signals allows the experimental identification of the laser-induced modification time range dependency on the laser parameters (see Fig. 2 (b), 4 (b)).

In Fig. 4 the experimentally determined time range was compared with the theoretically predicted liquid phase life time using a thermodynamic approach (see Eq. 2).

The experimentally determined modification time is almost similar to the liquid phase life time at low laser fluences. However, at higher laser fluences $\Phi>400 \mathrm{~mJ} / \mathrm{cm}^{2}$ the theoretically predicted liquid phase life time is distinct higher than the experimentally determined modification time.

The transmission/reflection variation is mainly induced by the deformation of the metal layer and the deformation only occurs in the liquid phase state. That means, the experimentally determined modification time is defined by two different effects: the life time of the laser-induced liquid phase and the transformation time, especially the time which is necessary for the formation of metal droplets.

At low laser fluences, the single pulse irradiation causes an incomplete metal droplet formation process (e.g. Fig. 3), that means, the experimentally determined modification time is mainly defined by the liquid phase life time. At high laser fluences, the single pulse irradiation results in the formation of metal droplets where in same cases residual chromium forms additional structures like metal bars. The additional components removed by further irradtion, see Fig. 2.

That means the experimentally determined modification time is mainly defined by the transformation time. Hence, the theoretically estimated liquid phase life time exceeds the experimentally determined transformation time at high laser fluences (see Fig. 4 (b)). It is noticed that the decoupled approach regarding the dewetting process (separated calculation of the heat and Navier-Stokes equation) and non-regarding further energy loss mechanisms can also result in differences between the experimental results and the theoretically predicted values.

For the estimation of the second limitation of the modification time - the transformation time - the considering of the time-dependent hole expansion process (see Eq. 6a and Fig. 5) using the Navier-Stokes equation (see Eq. 4) as well as the time-dependent transmission signal is necessary.

The time-dependent transmission signal can be described by the ratio between the dewetting-induced exposed fused silica areas and the metal-covered areas. Based on the theoretically predicted behaviour of the liquid phase, the transmission $\mathrm{T}$ dependent on the laser parameters $\Phi$ and $\mathrm{N}$ and on the time $\mathrm{t}$ can be estimated by:

$$
T(\Phi, N, t) \approx\left\{\begin{array}{cc}
\frac{\pi \cdot d_{h}^{2}\left(t+\Delta t_{L Q}^{C r}(\Phi) \cdot(N-1)\right)}{4 \cdot \Lambda^{2}}+T_{0} \quad \text { for } \quad 0 \leq t<\Delta t_{L Q}^{C r}(\Phi) \\
\frac{\pi \cdot d_{h}^{2}\left(N \cdot \Delta t_{L Q}^{C r}(\Phi)\right)}{4 \cdot \Lambda^{2}}+T_{0} \quad \text { for } \quad t \geq \Delta t_{L Q}^{C r}(\Phi)
\end{array}\right.
$$


with Eq. 3 and $6 b, \Lambda$ : average hole distance of the centre point and $\mathrm{T}_{0}$ : transmission signal through the $20 \mathrm{~nm}$ chromium. For the used red diode laser radiation the absorption coefficient of the chromium is $\alpha=6.36 \cdot 10^{7} \mathrm{~m}^{-1}$, that means the relative transmission signal through $20 \mathrm{~nm} \mathrm{Cr}$ is $\mathrm{T}_{0} \sim 30 \%$.

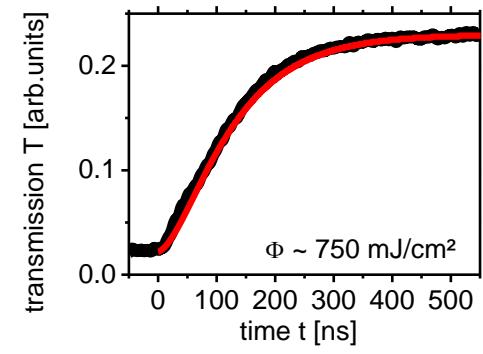

(a)

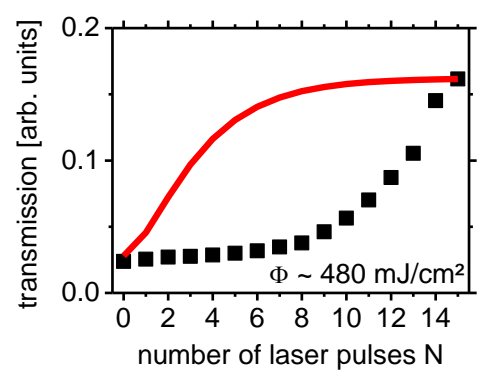

(c)

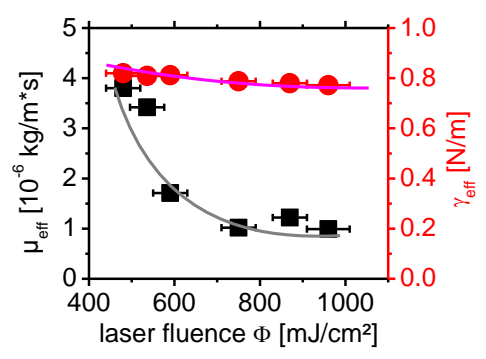

(b)

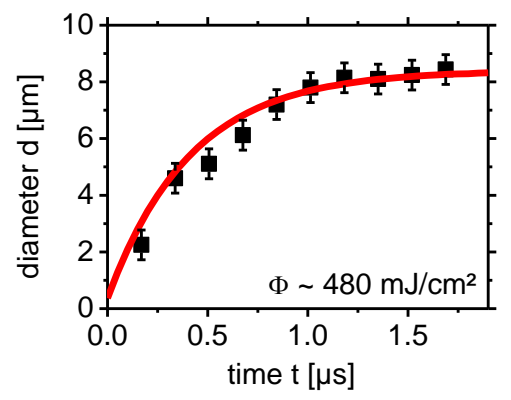

(d)

Fig. 6 (a) (black line) Measured transmission signal in dependence on the time at $\Phi=(750 \pm 50) \mathrm{mJ} / \mathrm{cm}^{2}$ and $\mathrm{N}=1$, (red line) fitted curve using Eq. 7 under the assumption of an effective viscosity $\mu_{\text {eff }}=0.88 \cdot 10^{-6} \mathrm{~kg} / \mathrm{m} \cdot \mathrm{s}$ and an effective surface tension $\gamma_{\text {eff }}=0.788 \mathrm{~N} / \mathrm{m}$

(The red line was fitted under the assumption of a complete droplet formation process, under the assumption of an incomplete droplet formation process $\mu_{\text {eff }}=1.02 \cdot 10^{-6} \mathrm{~kg} / \mathrm{m} \cdot \mathrm{s}, \gamma_{\text {eff }}=0.788 \mathrm{~N} / \mathrm{m}$ ).

(b) Calculated effective viscosity $\mu_{\text {eff }}$ and surface tension $\gamma_{\text {eff }}$ at $N=1$ dependent on the laser fluence (magenta and grey line: demonstrate the trend).

(c) (black square) Measured transmission dependent on the number of laser pulses at $\Phi=(480 \pm 40) \mathrm{mJ} / \mathrm{cm}^{2}$, (red line) estimated T(N) dependency under the assumption of a liquid phase life time of $\Delta t_{L F, \text { eff }} \approx 169 \mathrm{~ns}$, of an effective viscosity $\mu_{\text {eff }}=3.8 \cdot 10^{-6} \mathrm{~kg} / \mathrm{m} \cdot \mathrm{s}$, and an effective surface tension $\gamma_{\text {eff }}=0.82 \mathrm{~N} / \mathrm{m}$.

(d) (black square) Measured hole diameter at $\Phi=(480 \pm 40) \mathrm{mJ} / \mathrm{cm}^{2}$ dependency on the time $t$ with $t=N \cdot \Delta t_{L F}$ under the assumption of a liquid phase life time of $\Delta t_{L F, \text { eff }} \approx 169 \mathrm{~ns}$, (red line) estimated hole diameter with Eq. 6 a (right side) under the assumption of an effective viscosity $\mu_{\text {eff }}=3.8 \cdot 10^{-6} \mathrm{~kg} / \mathrm{m} \cdot \mathrm{s}$ and an effective surface tension $\gamma_{\text {eff }}=0.82 \mathrm{~N} / \mathrm{m}$.

The theoretically deduced transmission and hole diameter function allows a description of the experimental results. For example, the transmission signal at $\Phi=750 \mathrm{~mJ} / \mathrm{cm}^{2}$ and $\mathrm{N}=1$ can be very well described with Eq. 7 (see Fig. $6(\mathrm{a})$ ) under the assumption of effective material parameters: $\mu_{\text {eff }}=0.88 \cdot 10^{-6} \mathrm{~kg} / \mathrm{m} \cdot \mathrm{s}$ and $\gamma_{\text {eff }}=0.788 \mathrm{~N} / \mathrm{m}$ and under the assumption of a complete droplet formation process at the first pulse. However, based on the SEM measurements (see Fig. 2) we know that two pulses are necessary for a complete droplet formation process, regarding the multi-pulse process effective parameters of $\mu_{\text {eff }}=1.02 \cdot 10^{-6} \mathrm{~kg} / \mathrm{m} \cdot \mathrm{s}$ and $\gamma_{\mathrm{eff}}=0.788 \mathrm{~N} / \mathrm{m}$ can be estimated.

Due to the decoupling consideration of the laser - solid (Eq. 2) interaction and the mass transport in the liquid (Eq. 4) the usage of effective material parameters for the estimation of the temperature-dependent material parameters in Eq. 4 is necessary.

In Fig. 6 (b) the effective viscosity $\mu_{\text {eff }}$ and surface tension $\gamma_{\text {eff }}$ dependent on the laser fluence $\Phi$ are shown. Both effective parameters decrease with increasing laser fluence. This effect can be physically explained by the temperature - laser fluence dependency (see Fig. 4 (a)). The average temperature in the liquid phase increases with increasing laser fluence (see Fig. 4 (a)).

The surface tension can be described by: $\gamma=\mathrm{a}-\mathrm{b} \cdot\left(\mathrm{T}-\mathrm{T}_{\mathrm{m}}\right)(\mathrm{a}=1.7 \mathrm{~N} / \mathrm{m}, \mathrm{b}=0.00032 \mathrm{~N} /(\mathrm{m} \cdot \mathrm{K})$ : material parameters, $\mathrm{T}_{\mathrm{m}}$ : melting point chromium) [40]. That means, a higher temperature $\mathrm{T}_{\text {eff }}$ results in a lower effective surface tension. The effective surface tension is slightly smaller than the estimated surface tension $\gamma\left(T_{\text {ave }}{ }^{\text {th }}\right)$ of $1.18 \mathrm{~N} / \mathrm{m}$ under the assumption of an average temperature $\mathrm{T}_{\text {ave }}{ }^{\text {th }}\left(750 \mathrm{~mJ} / \mathrm{cm}^{2}\right)=3820 \mathrm{~K}($ see Fig. 4 (a)). 
However, due to the distinct non-linear dependency of the transmission (Eq. 7) and the diameter function (Eq. 6b) on the temperature (see Eq. 6a with Eq. 5a/b taking into account the $\gamma(\mathrm{T})$ and $\mu(\mathrm{T})$ dependency) it is obvious that the value of the effective temperature $T_{\text {eff }}$ is not equal to the value of the average temperature $T_{\text {ave }}$. The viscosity is distinctly dependent on the temperature, too. The viscosity can be described by $\mu \sim \mathrm{T}^{1 / 2} \cdot \exp (\mathrm{E} / \mathrm{kT})$ [40]. That means, the estimated viscosity - temperature dependency from Kaptay [41] predicts that the viscosity decreases with increasing laser fluence in agreement with the found behaviour of the effective viscosity.

Furthermore, the calculated viscosity for chromium [41] at the temperature in the liquid phase (according [41] $\left.\mu(3820 \mathrm{~K})=7.44 \cdot 10^{-6} \mathrm{~kg} / \mathrm{m} \cdot \mathrm{s}\right)$ is in the same order of magnitude than the assumed effective viscosity from approx. $1 \cdot 10^{-6}$ to $4 \cdot 10^{-6} \mathrm{~kg} / \mathrm{m} \cdot \mathrm{s}$.

In addition, also the transmission $\mathrm{T}$ - number of laser pulses $\mathrm{N}$ dependency can be described by Eq. 7. For example in Fig. 6 (c), the T(N)-dependency at $480 \mathrm{~mJ} / \mathrm{cm}^{2}$ is shown and the experimentally found results can be described by Eq. 7 with a moderate conformity using $\mu_{\text {eff }}=3.8 \cdot 10^{-6} \mathrm{~kg} / \mathrm{m} \cdot \mathrm{s}$ and $\gamma_{\text {eff }}=0.82 \mathrm{~N} / \mathrm{m}$ under the assumption of an effective liquid phase life time of $\Delta \mathrm{t}_{\mathrm{LF}, \text { eff }}=169 \mathrm{~ns}$ (equal to the experimentally found value see Fig. 4 (b)). Furthermore, the same effective parameter allows a very good description of the measured hole diameter d - time t dependency at $480 \mathrm{~mJ} / \mathrm{cm}^{2}$ (see Fig. $6(\mathrm{~d})$ ). Due to the very small variation of the transmission signal at low laser fluences and at low numbers of laser pulses (see Fig. 3 (c)), the T(t) dependency is not suitable for analyses using Eq. 7.

The transmission signal dependent on the time $\mathrm{T}(\mathrm{t})$ for single pulses especially at the first pulse (see Fig. 6 (a)) can be described by Eq. 7 very well, where the multi-pulse dependency T(N) (see Fig. $6(\mathrm{c})$ ) can be described with moderate results. This effect can most likely be explained by the decoupling adoption of the laser-solid interaction and the mass transport in the liquid. At the first pulse the laser radiation is absorbed by the homogeneous and flat metal film and the movement of the liquid can be excluded for the simulation during the irradiation. That means, the decoupling approach for $\mathrm{T}(\mathrm{t})$ at the first pulse works very well.

However, for multi-pulse irradiation a distinct modification of the metal layer topography can be found (see Fig. 3 (b)). That means, the $\mathrm{T}(\mathrm{N})$ estimation using the decoupling approach and the assumption of a homogeneous metal layer in the laser-solid interaction will most likely result in distinct differences to the measured $\mathrm{T}(\mathrm{N})$.

The next expedient extension of the simulation will be the consideration of the laser radiation by the number of laser pulses dependent on the metal layer topography. This extension will most likely improve the results. However, the absorption of the laser radiation by a distinct metal structure induces a distinct temperature profile and due to a temperature-dependent material parameter the temperature distribution results in a mass flow. That means the coupling of the heat equation (laser-solid interaction and heat diffusion) with the Navier-Stokes equation is necessary to describe the physical effects.

Beside the time dependency, the usage of effective parameters also allows the estimation of the geometry of the resultant structures. The resultant droplet size can be estimated by [42]:

$$
D=\left[\frac{192 \cdot \pi^{2} \cdot \gamma}{A_{H}}\right]^{1 / 3} \cdot h^{5 / 3} .
$$

That means, under the assumption of effective material parameters a droplet size of $\sim 2 \mu \mathrm{m}$ for low $\left(480 \mathrm{~mJ} / \mathrm{cm}^{2}\right)$ and high laser fluences $\left(750 \mathrm{~mJ} / \mathrm{cm}^{2}\right)$ can be estimated. The theoretically calculated droplet size is higher than the experimentally found values of $\sim 0.9 \mu \mathrm{m}$ (see Fig. 2 and 3). This difference can most likely be explained by a laser-induced minor partial evaporation of the chromium. The evaporation effect is distinctly visible at high numbers of laser pulses (see Fig. 2 (a)). At $\Phi=750 \mathrm{~mJ} / \mathrm{cm}^{2}$ and $\mathrm{N}=10$ a small reduction of the droplet density and droplet size and at $\mathrm{N}=100$ a complete removal of the chromium can be detected. 


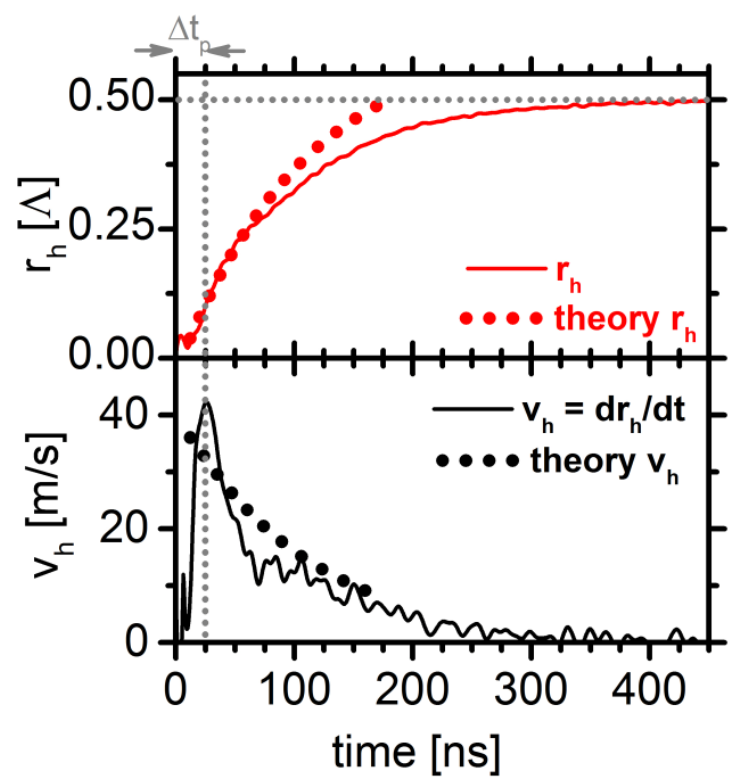

Fig. 7 (red line) Estimated hole radius $r_{h}$ from the transmission signal at $\Phi=750 \mathrm{~mJ} / \mathrm{cm}^{2}$ (see Fig. $\left.2(\mathrm{~b})\right)$ using Eq. 7 in units of $\Lambda$ (average hole distance of the centre points),

(red dots) theoretically predicted $r_{h}$ using Eq. 6(a) under the assumption of $\mu_{\text {eff }}=0.88 \cdot 10^{-6} \mathrm{~kg} /(\mathrm{m} \cdot \mathrm{s})$ and $\gamma_{\text {eff }}=0.788 \mathrm{~N} / \mathrm{m}(\mathrm{see}$ Fig. $6 \mathrm{~b})$,

(black line) velocity $v_{h}$ of the hole expansion $\left(v_{h}=d r_{h} / d t\right)$ with $\Lambda=6.6 \mu \mathrm{m}$,

(black dots) theoretically predicted velocity using the derivation of Eq. 6(a).

Beside the estimation of the liquid phase life time, the optical measurement allows also the estimation of the time-dependent hole radius $r_{h}(t)$ as well as the velocity of the hole expansion $v_{h}(t)=d_{h} / d t$. Under the approach that the hole expansion process is finished after the first pulse at high laser fluences like $\Phi=750 \mathrm{~mJ} / \mathrm{cm}^{2}(\mathrm{see}$ SEM Fig. 2 (a)) and that the resultant droplet diameter is distinct smaller than the average hole distance of the centre points, it is: $r_{h}(t \rightarrow \infty)=\Lambda / 2$. Based on this approach the $t_{h}(t)$ dependency can be estimated from the $T(t)$ measurement using Eq. 7. In Fig. 7 (red line) the $r_{h}(t)$ in units of $\Lambda$ is shown for $N=1$ and a laser fluence of $\Phi=750 \mathrm{~mJ} / \mathrm{cm}^{2}$. The dependent transmission measurement is shown in Fig. 6 (a). Taking into account the effective material parameters for $\Phi=750 \mathrm{~mJ} / \mathrm{cm}^{2}$ (see Fig. 6 (b)), a $\Lambda$ of $6.6 \mu \mathrm{m}$ can be estimated. Furthermore, the derivation of $r_{h}(t)$ taking into account the estimated $\Lambda$ allows the calculation of the velocity of the hole expansion and of the metal edge, respectively. The velocity increases during the irradiation and directly after the laser pulse a maximum velocity of $\sim 40 \mathrm{~m} / \mathrm{s}$ can be determined. After the irradiation the velocity decreases. The red dots represent the theoretically predicted $r_{h}(t)$ dependency using Eq. 6a taking into account the effective material parameters where the starting point of the hole opening process was set $10 \mathrm{~ns}$ after the laser pulse to improve the agreement between theory and experiment and considering the heating up of the metal.

At the beginning of the transformation process up to $\sim 75 \mathrm{~ns}$ after the start of the laser pulse the theory presents a good agreement with the experimentally estimated $\mathrm{r}_{\mathrm{h}}(\mathrm{t})$ behaviour.

After that a distinct divergence between the theory and the experimental results can be found. After the laser pulse a cooling down of the liquid film (see Fig. 4 (a)) results in an increasing of the viscosity of the liquid film and, finally, in a deceleration of the hole expansion. However, this effect is not included in the theoretical prediction, where a constant effective temperature was assumed and can explain the differences between the theory and the experimental results. Further, due to the non-observance of the cooling down the theoretically predicted expansion time $t_{\mathrm{s}}^{\text {th }}=\mathrm{t}_{\mathrm{s}}^{\text {th }}\left(\mathrm{r}_{\mathrm{h}}=\Lambda / 2\right)$ is $\mathrm{t}_{\mathrm{s}}^{\text {th }} \approx 175 \mathrm{~ns}$ and distinct smaller than experimental value of $\sim 375$ ns.

That means, the decoupled approach of the process (separated regarding of the heat and Navier-Stokes equations) results in an underestimation of the real metal droplet formation time (see Fig. 7). The next logical step for a better theoretical description of the process is a coupling of the both differential equations.

Furthermore, the derivation of the theoretical $r_{h}(t)$ curve allows the prediction of the velocity $v_{h}(t)$ (see Fig. 7; black dots: first derivation of the theoretically predicted $\mathrm{r}_{\mathrm{h}}(\mathrm{t})$ ).

The theoretical $v_{h}(t)$ curve does not describe the experimentally found behaviour very well. Especially the theory curve does not include the increasing of the velocity during the irradiation. Furthermore, at the beginning of the hole expansion process the theoretically predicted velocity is smaller up to $\mathrm{t} \sim 50 \mathrm{~ns}$ and after that the predicted velocity is above the experimentally estimated values.

\section{Conclusions and Outlook}


The dynamics as well as the resulting structures of the laser-induced nanostructuring by means of nanosecond laser pulse irradiation of a thin chromium layer on fused silica were studied. The results of the time-resolved optical transmission measurements clearly show that in addition to the finally achieved morphology of the nanostructured thin film, the dynamics of the processes depend on the laser parameters $(\Phi, \mathrm{N})$, too.

The experimental results (e.g., liquid phase life time and hole expansion velocity) were compared with a theoretical model, where the process was estimated under the assumption of a decoupling of the laser - solid interaction and the mass transport in the liquid.

The experimental results can be well simulated by the theoretical approach using laser fluence dependent effective material parameter. Hence, an optimization of process parameters based on the simulation can be initiated at this stage.

However, some effects like the hole radius dependent on the time can be limited described by the theoretical approach. Therefore, the coupling of both differential equation systems is most likely helpful to improve the significance and reliability of the results. Hole nucleation processes should be included into the model and may improve the hole formation simulation.

Furthermore, the proposed mechanism does not consider all possible contributions to the thermal and hydrodynamic processes but includes the most important ones from the current understanding. A more detailed model should consider additional processes. For the heat exchange of the system such processes are the energy dissipation by ablation or evaporation products, the heat loss by electromagnetic thermal radiation, the generation of mechanical energy due to acoustic waves, or mechanical processes as well as heating. For the energy redistribution of internal energy of the system the nucleation of holes in the film, the surface energy alteration due to dewetting, and viscosity changes can be considered. In addition, chemical reactions such as decomposition, oxidation, or structural changes such as crystallization can contribute to the heat balance.

Further, it can be expected that the simulation may allow the prediction of metal nanostructured patterns induced by a melting and transformation process. In particular, this method may allow the prediction of parameters required for the fabrication of desired nanostructures by laser-induced nanostructuring of thin films. After further refinements it can be applied for the production of periodic sub- $\mu \mathrm{m}$ metal structures using periodic laser beam profiles [42] or even more complex sub- $\mu \mathrm{m}$ structures, e.g. doughnut structures for infrared applications, by locally tuning the processing parameters.

In the future, the simulation has to be tested and verified with different metal/dielectric systems.

\section{Acknowledgements}

We are grateful for funding from the German Research Foundation (DFG: Deutsche Forschungsgemeinschaft) under LO 1986/2-1 and the German Academic Exchange Service under 56266271 (DAAD: Deutscher Akademischer Austauschdienst).

\section{References}

[1] B. Tan, K. Venkatakrishnan, J. Micromech. Microeng 16 (2006) 1080.

[2] M. Schade1, O. Varlamova, J. Reif, H. Blumtritt, W. Erfurth, H. S. Leip

[3] Y. Yang, J. Yang, L. Xue, Y. Guo, Appl. Phys. Lett. 97 (2010) 141101.

[4] R. LeHarzic, Optics Express 13 (2005) 6651.

[5] A. V. Vorobyev, C. Guo, Optics Express 14 (2006) 2164

[6] I. N. Zavestovskaya, Quantum Electron. 40 (2010) 942.

[7] A. Pereira, A. Cros, P. Delaporte, S. Georgiou, A. Manousaki, W. Marine, M. Sentis, Appl. Phys. A 79 (2004) 1433.

[8] S. Imamova, A. Dikovska, N. Nedyalkov, P. Atanasov, M. Sawczak, R. Jendrzejewski, G. Śliwiński, M. Obara, J. Optoelectron. Adv. Mater. 12 (2010) 500.

[9] S. J. Henley, C. H. P. Poa, A. A. D. T. Adikaari, C. E. Giusca, J. D. Carey, S. R. P. Silva, Appl. Phys. Lett. 84 (2004) 4035

[10] K. Ratautas, M. Gedvilas, G. Račiukaitis, A. Grigonis, J. Appl. Phys. 112 (2012) 013108.

[11] P. R. Herman, R. S. Marjoribanks, A. Oettl, K. Chen, I. Konovalov, S. Ness, Appl. Surf. Sci. 154-155 (2000) 577.

[12] M. Lenzner, J. Krüger, W. Kautek, F. Krausz, Appl. Phys. A 68 (1999) 369

[13] J. Ihlemann, B. Wolff, P. Simon, Appl. Phys. A 54 (1992) 363.

[14] J. Ihlemann, B. Wolff-Rottke, Appl. Surf. Sci. 106 (1996) 282.

[15] R. Böhme, K. Zimmer, D. Ruthe, B. Rauschenbach, J. Laser Micro/Nanoeng. 1 (2006) 190.

[16] K. Zimmer, R. Böhme, C. Vass, B. Hopp, Appl. Surf. Sci. 255 (2009) 9617.

[17] M. Ehrhardt, G. Raciukaitis, P. Gecys, K. Zimmer, Appl. Phys. A 101 (2010) 399.

[18] P. Lorenz, M. Ehrhardt, K. Zimmer, Phys. Status Solidi A 209 (2012) 1114.

[19] E. Matthias, M. Reichling, J. Siegel, O. W. Käding, S. Petzoldt, H. Skurk, P. Bizenberger, E. Neske, Appl. Phys. A 58 (1994) 129.

[20] S. J. Henley, J. D. Carey, S. R. P. Silva, Phys. Rev. B 72 (2005) 195408.

[21] D. Bäuerle, Laser Processing and Chemistry, 3rd Ed., Springer, Berlin, Heidelberg, New York, 2000.

[22] R. F. Wood, G. E. Giles, Phys. Rev. B 23 (1981) 2923.

[23] P. Lorenz, M. Ehrhardt, K. Zimmer, Appl. Surf. Sci. 258 (2012) 9138.

[24] P. Lorenz, M. Ehrhardt, K. Zimmer, Appl. Surf. Sci. 258 (2012) 9742.

[25] P. Lorenz, M. Ehrhardt, K. Zimmer, Proc. SPIE 8243 (2012) 82430 Y.

[26] P. Lorenz, M. Klöppel, F. Frost, M. Ehrhardt, K. Zimmer, P. Li, "Laser-induced circular nanostructures in fused silica assisted by a self-assembling chromium layer", Appl. Surf. Sci. 280 (2013) 933. 
[27] P. Lorenz et al., Appl. Phys. A 111 (2013) 1025-1030.

[28] A. Sharma, R. Khanna, Phys. Rev. Lett. 81 (1998) 3463.

[29] A. Yochelis, E. Knobloch, L. M. Pismen, Eur. Phys. J. E 22 (2007) 41.

[30] A. Vrij, Discuss. Faraday Soc. 42 (1966) 23.

[31] Ch. Favazza, R. Kalyanaraman, R. Sureshkumar, Nanotechnology 16 (2006) 4229

[32] S. J. Henley, J. D. Carey, S. R. P. Silva, Appl. Surf. Sci. 253 (2007) 8080

[33] D. A. Willis, X. Xu, J. Heat Transfer 122 (2000) 763.

[34] J. Trice, D. Thomas, Ch. Favazza, R. Sureshkumar, R. Kalyanaraman, Phys. Rev. B 75 (2007) 235439.

[35] Z. Toth, B. Hopp, Z. Kantor, F. Ignacz, T. Szörenyi, Z. Bor, Appl.Phys. A 60 (1995) 431.

[36] H.M. Bulgakova, A.V. Bulgakov, L.P. Habich, Appl. Phys. A 79 (2004) 1323

[37] http://www.mt-berlin.com/frames_cryst/descriptions/quartz\%20.html, March 2014 .

[38] W.M. Haynes (Ed.), Handbook of Chemistry and Physics, 93rd edition, CRC Press, Taylor \& Francis Group, Boca Raton, London, New York, 2012.

[39] A. Sharma, E. Ruckenstein, Langmuir 2 (1986) 480.

[40] George Kaptay, "A unified equation for the viscosity of pure liquid metals", Z. Metallkd. 96 (2005) 1.

[41] Takamichi et al: The physical properties of liquid metals, Oxford Calderon Press (1993).

[42] K. Ratautas et al., JLMN 7 (2012) 355

[43] M. Mäder, Th. Höche, J. W. Gerlach, R. Böhme, B. Rauschenbach, „Nanostructures by diffraction mask projection laser ablation“, Phys. Status Solidi B 247 (2010) 1372. 